\title{
Compressed Sensing Techniques for Detecting Damage in Structures
}

\author{
David Mascareñas* ${ }^{1}$, Alessandro Cattaneo ${ }^{2}$, James Theiler ${ }^{3}$, Charles Farrar $^{1}$ \\ ${ }^{1}$ Engineering Institute, Los Alamos National Laboratory, P.O. Box 1663, MS T001 \\ Los Alamos, NM, 87544 \\ ${ }^{2}$ Politecnico di Milano, Department of Mechanics, Via La Masa 1, Milan, 20156 (MI), \\ Italy \\ ${ }^{3}$ ISR-3, Los Alamos National Laboratory, P.O. Box 1663, MS B244 Los Alamos, \\ NM, 87544 \\ *Corresponding Author, Work: 505-663-5227, Cell: 858-380-8648, Fax: 505663 5225, \\ dmascarenas@lanl.gov
}

\begin{abstract}
One of the principal challenges facing the structural health monitoring (SHM) community is taking large, heterogeneous sets of data collected from sensors, and extracting information that allows the estimation of the damage condition of a structure. Another important challenge is to collect relevant data from a structure in a manner that is cost effective, and respects the size, weight, cost, energy consumption, and bandwidth limitations placed on the system. In this work we established the suitability of compressed sensing to address both challenges.

A digital version of a compressed sensor is implemented on-board a microcontroller similar to those used in embedded SHM sensor nodes. The sensor node is tested in a surrogate SHM application using acceleration measurements. Currently the prototype compressed sensor is capable of collecting compressed coefficients from measurements and sending them to an off-board processor for signal reconstruction using $C_{\text {n }}$ norm minimization. A compressed version of the matched

filter known as the smashed filter, has also been implemented on-board the sensor node, and its suitability for detecting structural damage will be discussed.
\end{abstract}

\section{INTRODUCTION}

Data for structural health monitoring applications is generally collected using a distributed sensor network. Distributed sensor networks made up of nodes with hardwired data and communication lines are generally have high installation costs, particularly in the retrofit mode. Lynch reported that hard-wire installation could consume $75 \%$ of the total testing time, and installation costs could consume $25 \%$ of the total system costs [1]. The goal is to transition to low-power, wireless sensor networks featuring minimal installation costs [2]. Two of the major problems with these types of sensor networks are the minimization of energy and bandwidth. Compressed sensing techniques hold promise to help address both of these demands. By collecting compressed coefficients, the signal of interest can be represented using a fraction of the measurements required by traditional Nyquist sampling. The result is 
reduced energy consumption for data collection, storage and transmission [3], [4]. In addition, the bandwidth required to transmit the sampled signal is also significantly reduced. The focus of this work is to evaluate the applicability of compressed sensing techniques to expand the capabilities of wireless sensor networks for structural health monitoring applications.

\section{BACKGROUND OF COMPRESSED SENSING}

Compressed sensing has been a prolific research topic in applied math and statistics over the last few years. Excellent tutorials covering the basics of compressed sensing can be found in [5], [6], and [7]. To summarize, a signal of interest $x$ can be represented as:

$$
x=\sum_{i=1}^{N} s_{i} \psi_{i} \text { or in matrix form as } x=\Psi s
$$

Where $\Psi$ is an orthonormal basis and " $\mathrm{s}$ " is the representation of the signal in the $\Psi$ domain. In the case of compressed sensing we are interested in the case where $x$ is compressible in some domain. That is, the number of significant non-zero elements of $s$ is equal to $K$ and $K<<N . \quad K$ is known as the "sparsity" of the signal. A measurement matrix " $\Phi$ " is then introduced to produce compressed sensing coefficients $y$.

$$
y=\Phi x=\Phi \Psi s=\Theta s
$$

Where $\Phi$ has $M<<N$ rows. At this point it is important to note that this equation represents an underdetermined system of linear equations. One of the major breakthroughs of the compressed sensing community was the finding that assuming $K<<N$ it is possible to recover $x$ from $y$ assuming the matrix $\Phi$ possesses the restricted isometry property (RIP) and $x$ is sparse in some basis [5]. An example of a sparse signal would be a signal that only contains a few non-zero Fourier coefficients such as a sum of decreasing harmonics that represents a structure's impulse response. The direct formulation of this problem is finding the vector $s$ with minimal $C_{0}$ norm. Unfortunately $\ell_{0}$ norm minimization is numerically unstable and computationally expensive [7]. It has been shown though that the $\ell_{0}$ can be replaced with an $\ell_{\text {norm }}$ relaxation [6]. The $\ell_{\text {n }}$ norm regularization problem [5] [8] can be solved to recover sparse signals from the compressed coefficients $y$. Amazingly, it is possible to recover $x$ using an $M$ measurements following the relation [5]:

$$
M \geq c K \log \left(\frac{N}{K}\right)
$$

Where $c$ is a constant that has empirically been found to approximately equal 4.0 [7]. Equation (3) implies that it is possible to reconstruct a sparse signal $x$ using far fewer 
measurements than elements in $x$. In this work, the $\ell_{\text {norm regularization approach }}$

will be explored for recovering the signal $x$ from compressed coefficients $y$.

To date the literature contains few examples of compressed sensing techniques being applied to the structural health monitoring field. The earliest work found that exploited compressed sensing for SHM applications was that by Cortial [9]. Cortial applied compressed sensing techniques to a simulated structural health monitoring sensor network with a high spatial resolution installed on-board an F-16. Cortial found there was potential to use a subset of his distributed sensors to locate and identify damage to the F-16 caused by an explosive bullet when compressed sensing techniques were applied. More recently, Bao [10] applied compressed sensing techniques to acceleration data collected from the SHM system for the Shandong Binzhou Yellow River highway bridge. This work found that for a given signal reconstruction error, conventional techniques such as wavelet data compression enjoyed a higher compression ratio than compressed sensing applied using either the Fourier or wavelet basis. Despite the results presented by Bao, the author believes that compressed sensing techniques offer advantages that offset the compression ratio penalty when compared to conventional compression techniques. For instance, compressed coefficient are "democratic" in the sense that no one coefficient carries any more information than any other. This property could help enable the design of measurement systems that fail gracefully if the system experiences partial malfunctions. Compressed sensing also offers the possibility of enabling low-power measurement hardware. This advantage will be particularly important for longendurance applications such as those common in structural health monitoring applications. Furthermore, by applying techniques such as the over-complete dictionary the performance of compressed sensing for damage detection applications can be improved.

\section{EXPERIMENTAL SETUP}

In order to evaluate the applicability of compressed sensing for embedded structural health monitoring sensor nodes, a digital prototype of a compressed sensor node was built. The prototype consisted of an ATmega1281 microcontroller, an Integrated Circuit - Piezoelectric (ICP $\AA$ ) accelerometer, and the associated amplification and ICP circuitry required to interface the analog-to-digital converter (ADC) of the microcontroller to the ICP® accelerometer. The accelerometer was then attached to the second floor of a representative 3 story structure [11], [12] as shown in Figure 1. The accelerometer was oriented to measure the transverse vibration of the 3story structure. An electro-magnetic shaker was then attached to the base of the three story structure to provide a source of excitation. The excitation to the structure was a sine wave with a frequency of $30.7 \mathrm{~Hz}$ which corresponds to the first resonant frequency of this structure. In order to introduce damage into the structure, a bumper was used to induce a nonlinear response when the relative transverse displacement between the second floor and the base would exceed a threshold value. This nonlinearity could represent damage such as a crack opening and closing. The signal from the accelerometer was sampled by the ATmega1281 with a 10 bit ADC at a 
sampling rate of about $3000 \mathrm{~Hz}$. The sampling rate is significantly higher than the structural excitation frequency in order to capture the higher frequency dynamics caused by induced nonlinearities. In this work, 256 point time series were collected by the Atmega1281 and subsequently converted into compressed measurements by the microcontroller. The elements of the measurement matrix $\Phi$ were chosen to be either \pm 1 . The generation of the measurement matrix $\Phi$ was accomplished using a linear feedback shift register (LFSR) similar to that mentioned in [13]. The \pm 1 measurement matrix was selected in order to allow the generation of the compressed coefficients onboard the microcontroller using integer arithmetic. The ATmega1281 was placed onboard an STK500 evaluation board. A base station laptop was then connected to the STK500 in order to facilitate the debugging of the compressed sensing algorithms, and to expedite the collection of compressed coefficients from the embedded sensor node. For the purpose of this experiment, the ATmega1281 would transmit both the compressed measurements as well as the original signal when it was queried for a measurement. By collecting both pieces of data the reconstructed signal derived from the compressed coefficients could be compared to the original signal in order to evaluate the performance of compressed sensing techniques.

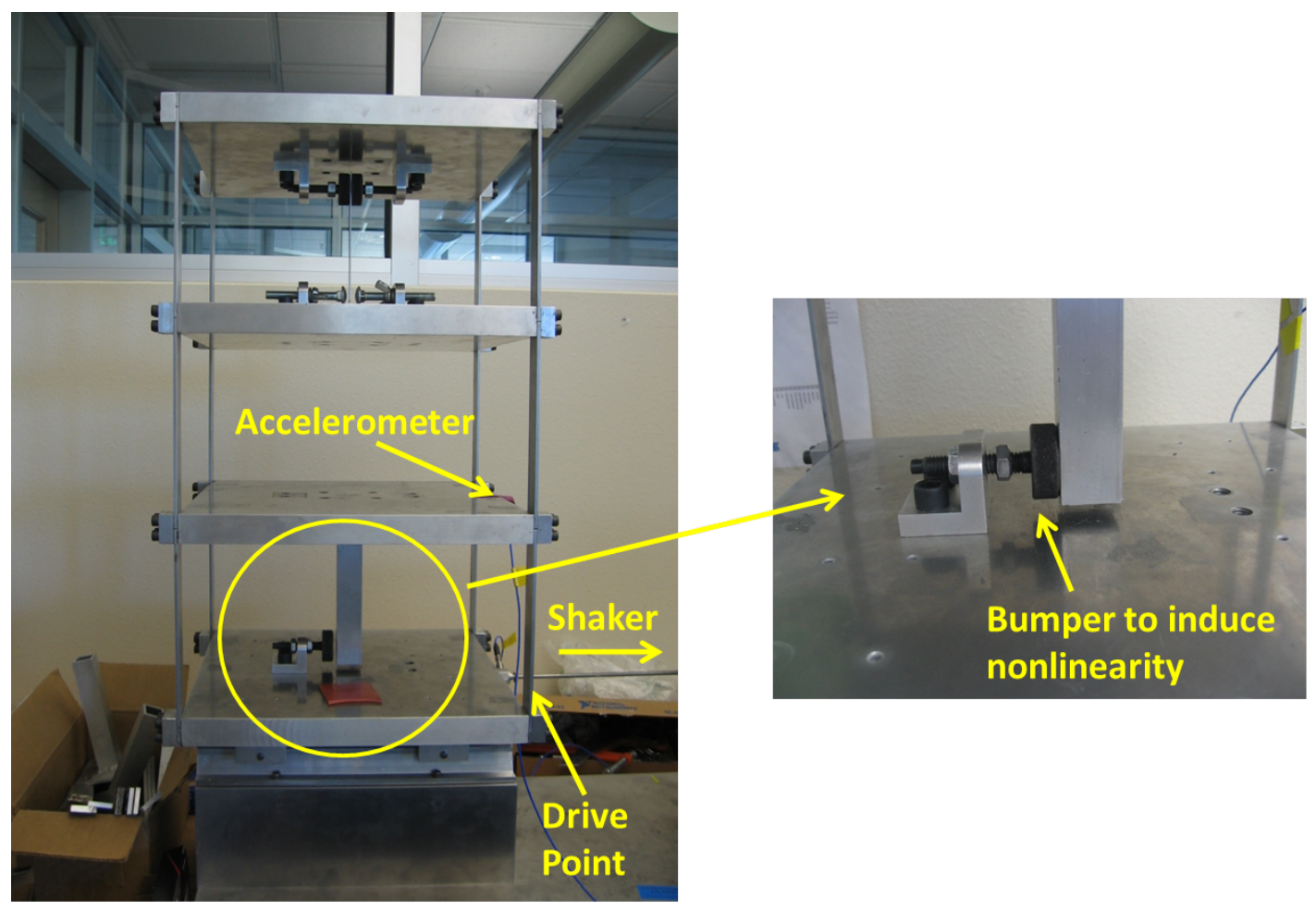

Figure 1- Representative 3 story structure used to evaluate the compressed sensor node.

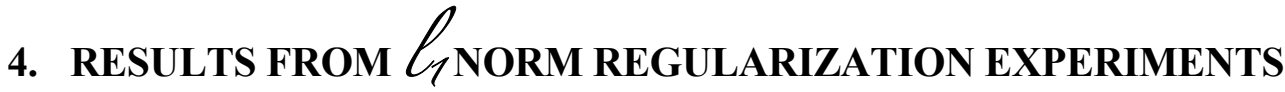

The structure described in the previous section was placed in a configuration so that it could assume either a damaged state or an undamaged state. In the undamaged state, the output from the accelerometer should assume a sine wave with a frequency of 30.7 $\mathrm{Hz}$ that corresponds to the shaker excitation frequency. In the damaged state, the 
bumper interacts with the structure and the frequency content of the resulting output from the structure is more widely distributed across the spectrum. For this work we assume that the resulting signals from the structure should be sparse in the Fourier basis. Data $x$ were collected from the structure in both the damaged and undamaged states, and were subjected to the compressed sensing measurement process to generate the compressed coefficients $y$.

$$
y=\Phi x
$$

Where $\Phi$ is an $M$ by $N$ matrix generated using linear feedback shift registers. The resulting elements of $\Phi$ take values of \pm 1 . $M$ indicates the number of compressed coefficients, and $N$ is the length of the original signal. The resulting compressed measurements were then cast into the $C_{\text {i }}$ norm regularization framework to attempt recovery of the original signal. The $C_{\text {norm }}$ regularization problem can be written as:

$$
\underset{s}{\operatorname{Minimize}}\left(\|y-\Phi \Psi s\|_{2}+\gamma\|s\|_{1}\right)
$$

In this work $\Psi$ was taken to be the Fourier basis. The $C_{\text {norm }}$ regularization problem

trades off between the size of the residual and the sparsity of $s$. The $C_{\text {n }}$ norm regularization was implemented using the CVXMOD software [14]. 256-point timeseries data were collected from the structure using the ATmega1281 and were transmitted to the base station laptop. The resulting compressed coefficients were

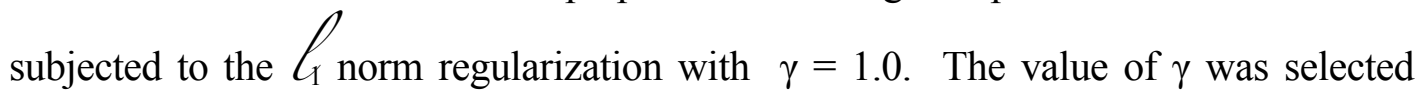
heuristically. The basis in which the signal is sparse, $\Psi$, is taken to be the Fourier basis. The $C_{1}$ norm regularization problem was solved using 16, 32, 64 and 128

compressed coefficients for both the damaged and undamaged cases. These correspond to using only $6.25 \%, 12.5 \%, 25 \%$, and $50 \%$ respectively of the original number of measurements. The resulting reconstructions can be found in Figure 2 and Figure 3. The values of the Fourier basis coefficients for the undamaged and damaged cases are found in Figure 4 and Figure 5, respectively.

By looking at the Fourier coefficient plots in Figure 4 and Figure 5 it can be seen that the undamaged signal has approximately 12 non-zero components, and the damaged signal has approximately 24 non-zero components. From this information it is expected that more compressed coefficients will be required to reconstruct the damaged signal as opposed to the undamaged signal. From the reconstructed signals in Figure 2 and Figure 3 we can see that the nature of the output signal does not begin to become apparent until about 64 compressed coefficients are taken. This result is to be expected because prior empirical results [7] suggest that the reconstruction should require about $4 * \mathrm{~K}$ coefficients, or about 48 coefficients in the undamaged case, and 96 
coefficients in the damaged case. From the plots we see the reconstructed signal captures the original signal fairly well once 128 compressed coefficients (half the original number of measurements) are used in the reconstruction.

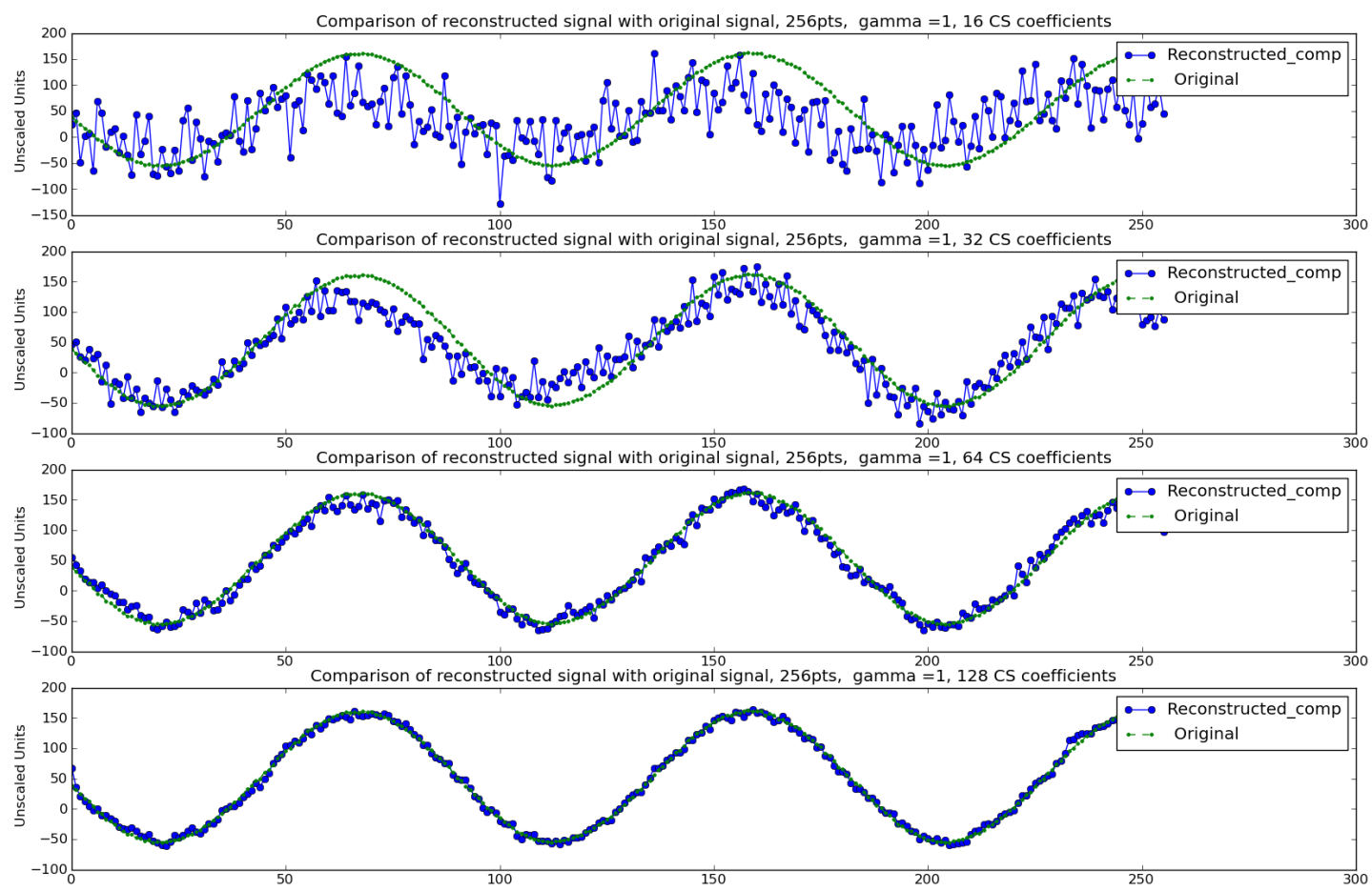

Figure 2 - Undamaged case compressed sensing reconstruction
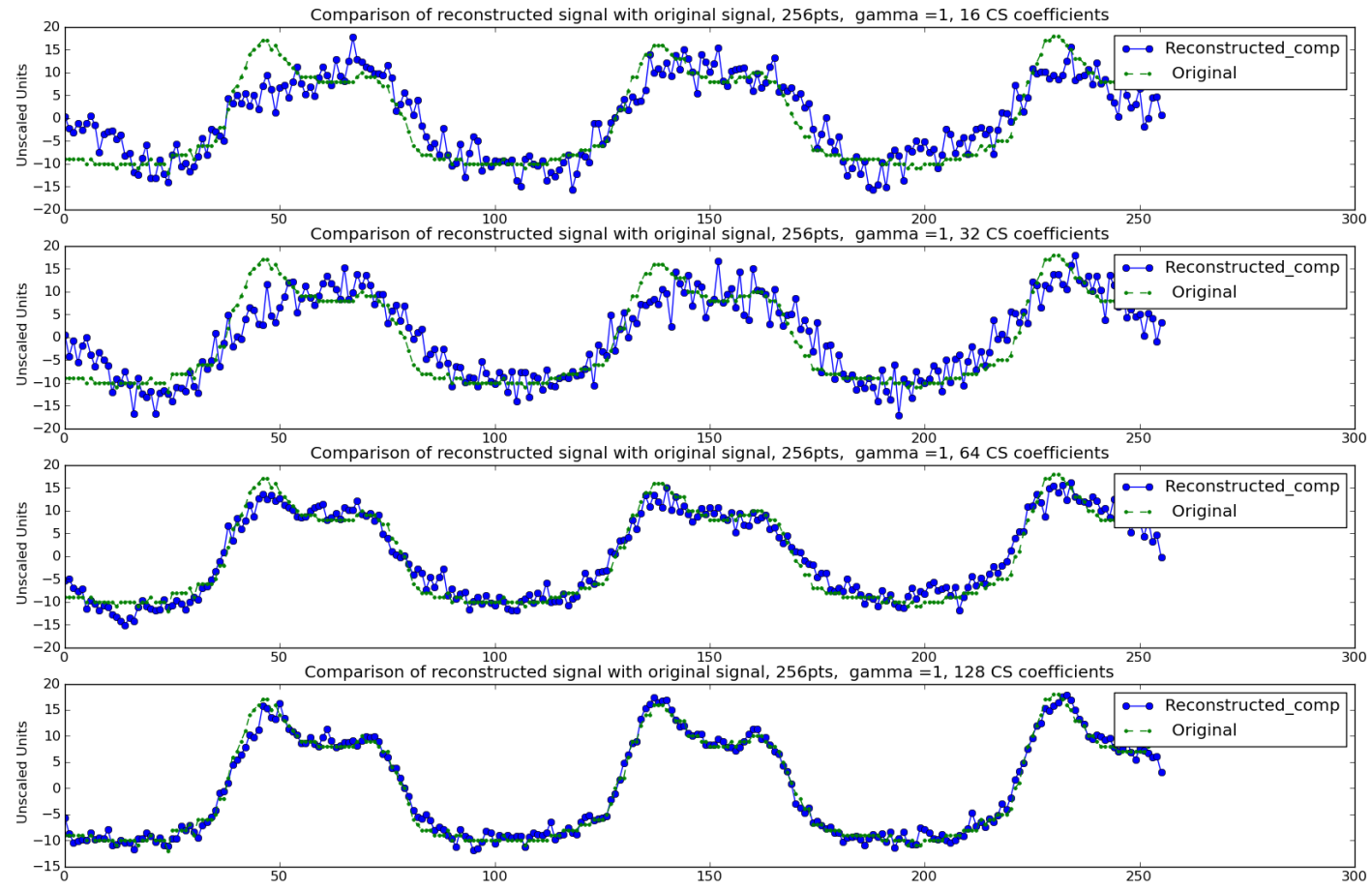

Figure 3 - Damaged case compressed sensing reconstruction

Mascareñas, D., Cattaneo, A., Theiler, J., Farrar, C., Los Alamos National Laboratory, PO Box 1663

MS T001, Los Alamos, NM 87544, LA-UR 12-01484 

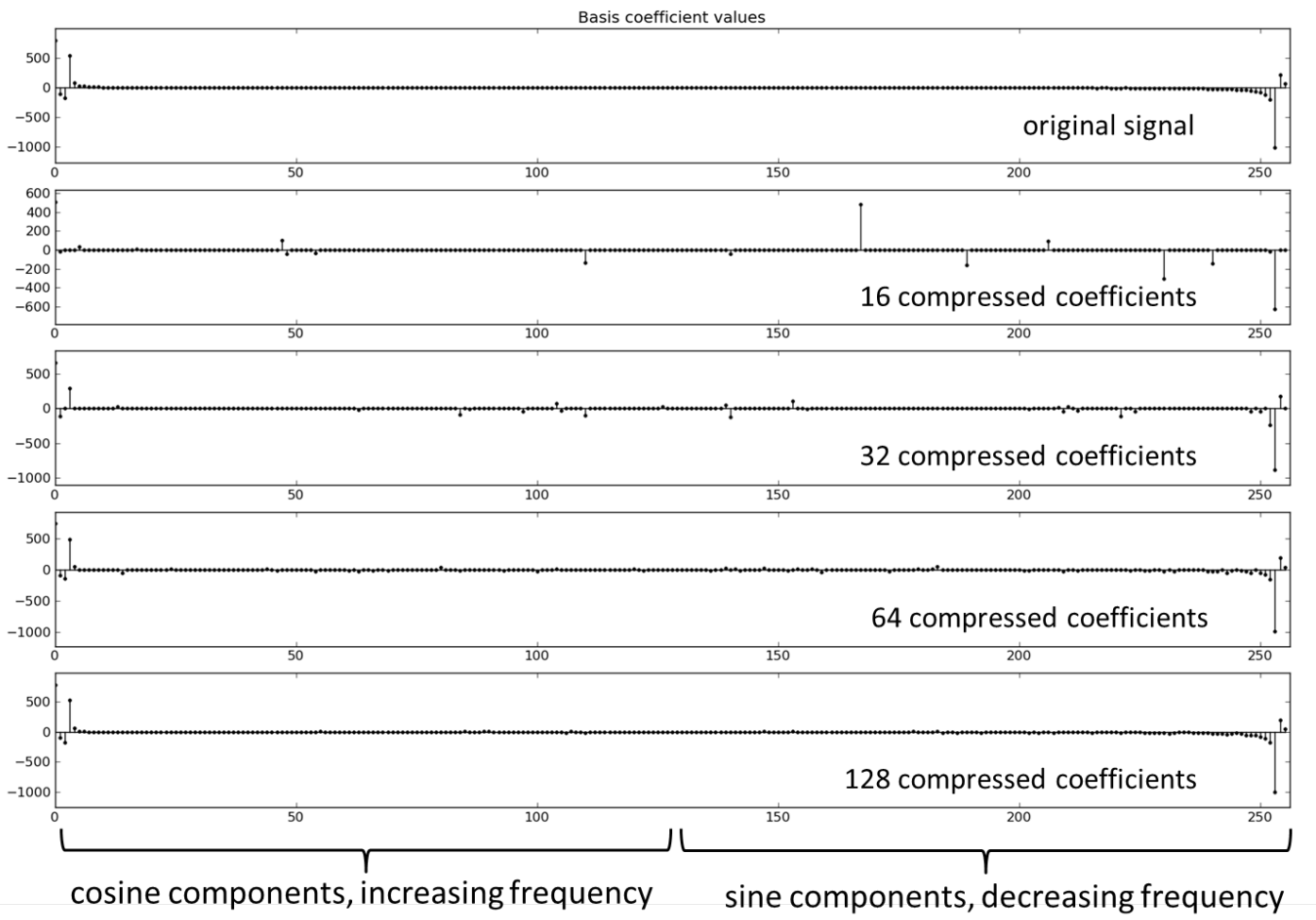

Figure 4 - Fourier basis coefficients from the healthy structure, original and reconstructed signals.

Next consider the plots of the Fourier basis coefficients displayed in Figure 4 and Figure 5. The first noteworthy aspect of these plots, is that the reconstructed signal features the most dominant peak in the Fourier basis coefficients even when only 16 compressed coefficients are used in the reconstruction. This result suggests that if only an estimate of the dominant frequency of a signal is required, it may be possible to produce an accurate estimate using only a very small number of compressed coefficients. At low numbers of compressed coefficients, spurious small but non-zero values of the basis coefficients appear across the coefficient vector. There is no immediately obvious bias as to how these non-zero, small components are distributed across the coefficient vector. As the number of compressed coefficients used is increased, the magnitude of these spurious components diminishes to 0 . In addition, as the number of compressed coefficients increases, the less dominant peaks in the Fourier basis coefficients begin to emerge. Once 128 compressed coefficients are used in the optimization, the Fourier basis coefficients of the original signal, and the reconstructed signal are very similar.

It is interesting to note, that in both the undamaged and damaged cases, the signal reconstructions seem to basically become less "fuzzy" as additional coefficients are included in the $C_{1}$ norm regularization process. As we use more compressed coefficients, the signal comes more into focus. This phenomenon illustrates the democratic property possessed by compressed coefficients. Compressed coefficients are democratic in the sense that no particular compressed coefficient carries any more 
information than any other. If any compressed coefficient were to be lost, it could simply be replaced by another compressed coefficient. Alternatively, if the coefficient could not be replaced, the resulting reconstructed signal would simply go slightly out of focus, but the main features of the signal would still be intact although not shown explicitly in this study. The democratic nature of compressed coefficients can be exploited to produce measurement systems that degrade gracefully with minor failures and losses in communication.

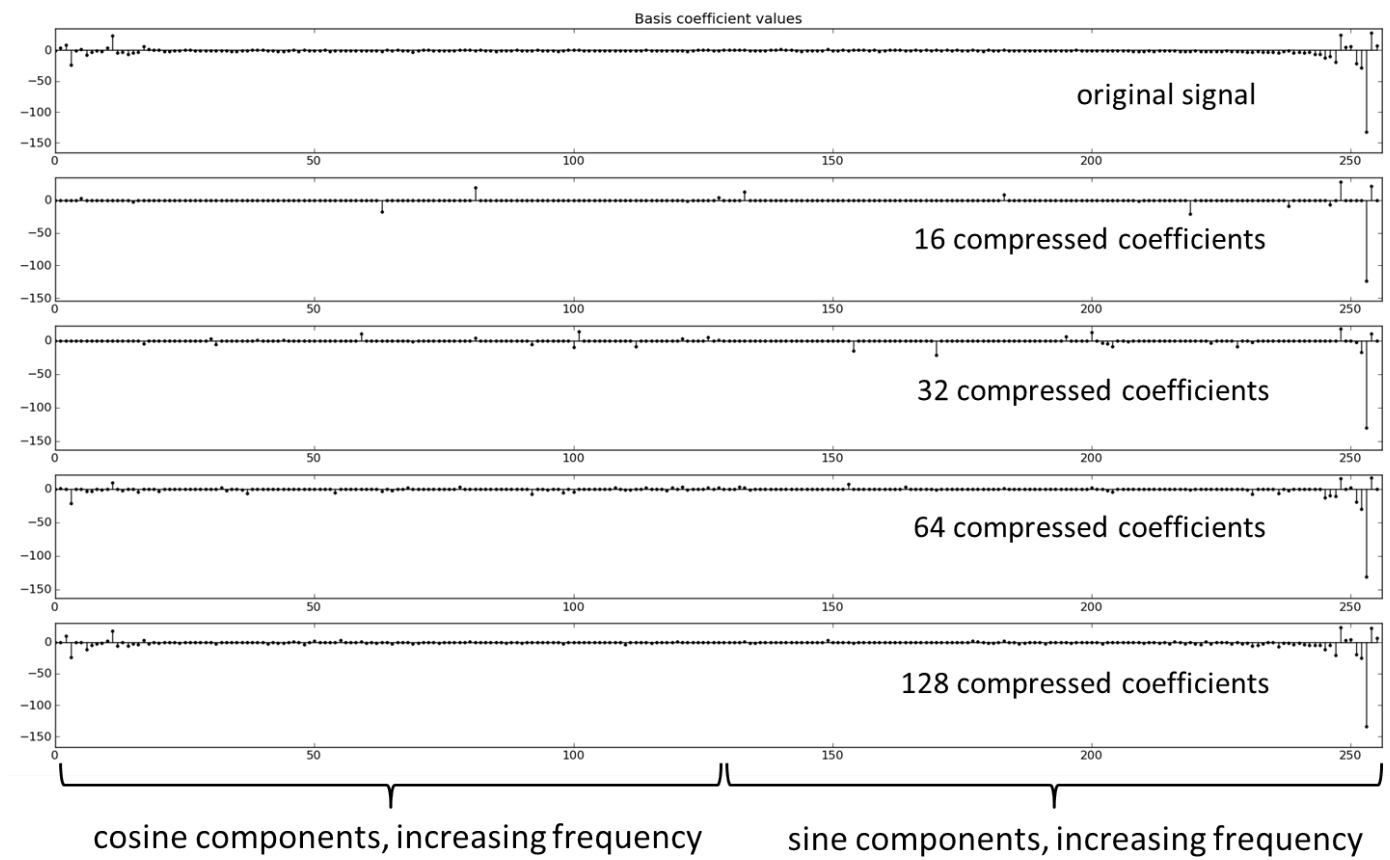

Figure 5 - Fourier basis coefficients from damaged structure, original and reconstructed signals.

\section{GRECONSTRUCTION WITH OVER-COMPLETE DICTIONARIES}

Oftentimes signals associated with structural health monitoring applications are generated by systems that are nearly linear being excited by oscillatory sources. As a result, it is natural to represent these signals in the Fourier domain using the discrete Fourier transform. Generally, the frequency content of the signals being measured does not exactly correspond to the frequencies being sampled by the discrete Fourier transform of the signal captured by the data acquisition. The result is the emergence of the "leakage" phenomenon commonly encountered in Fourier analysis [15]. Leakage effectively decreases the sparsity of a measured signal in the discrete Fourier basis. The underlying signal being sampled may only contain a single frequency component, but if its frequency is in-between the frequencies sampled by the discrete Fourier transform, it will manifest itself as non-zero content at many frequencies. The result is that the signal is less sparse in the discrete Fourier basis. One method for effectively regaining sparsity in the signal, is to use an over-complete dictionary for $\Psi$ instead of a basis. The term "over-complete dictionary" refers to a set of vectors that contains a complete set of basis vectors plus additional vectors not in the original basis set. Here the over-complete Fourier basis is used to reconstruct the signal [16]. The 
over-complete Fourier basis is generated by sampling the standard discrete Fourier basis more finely in frequency. The over-complete Fourier basis is generated by:

$$
\begin{gathered}
\psi(\omega, n)=\cos \left(\frac{2 \pi k n}{L N}\right) \text { for } k=0, \ldots, \frac{L N}{2} \\
\psi(\omega, n)=\sin \left(\frac{2 \pi k n}{L N}\right) \text { for } k=1, \ldots, \frac{L N}{2}-1 \\
\quad \text { for } n=0, \ldots, N-1
\end{gathered}
$$

Where $N$ is the length of the vectors, and $L$ is the multiplicative factor by which the dictionary is over-complete. For this work, $L$ is taken to be 2, so the over-complete dictionary contains 2 times as many vectors as the standard Fourier basis. The overcomplete dictionary basically includes the frequency components in-between those sampled by the standard Fourier basis. The $C_{1}$ norm regularization problem described by (4) was repeated for both the healthy and damaged signals. $\Psi$ was replaced with the over-complete Fourier dictionary, and $\gamma=1.0$. The $\ell_{1}$ norm regularization problem was solved using $8,16,32$, and 64 compressed coefficients for both the damaged and undamaged cases. The reconstruction of the healthy signal can be found in Figure 6 and the reconstruction of the damaged signal is found in Figure 7.

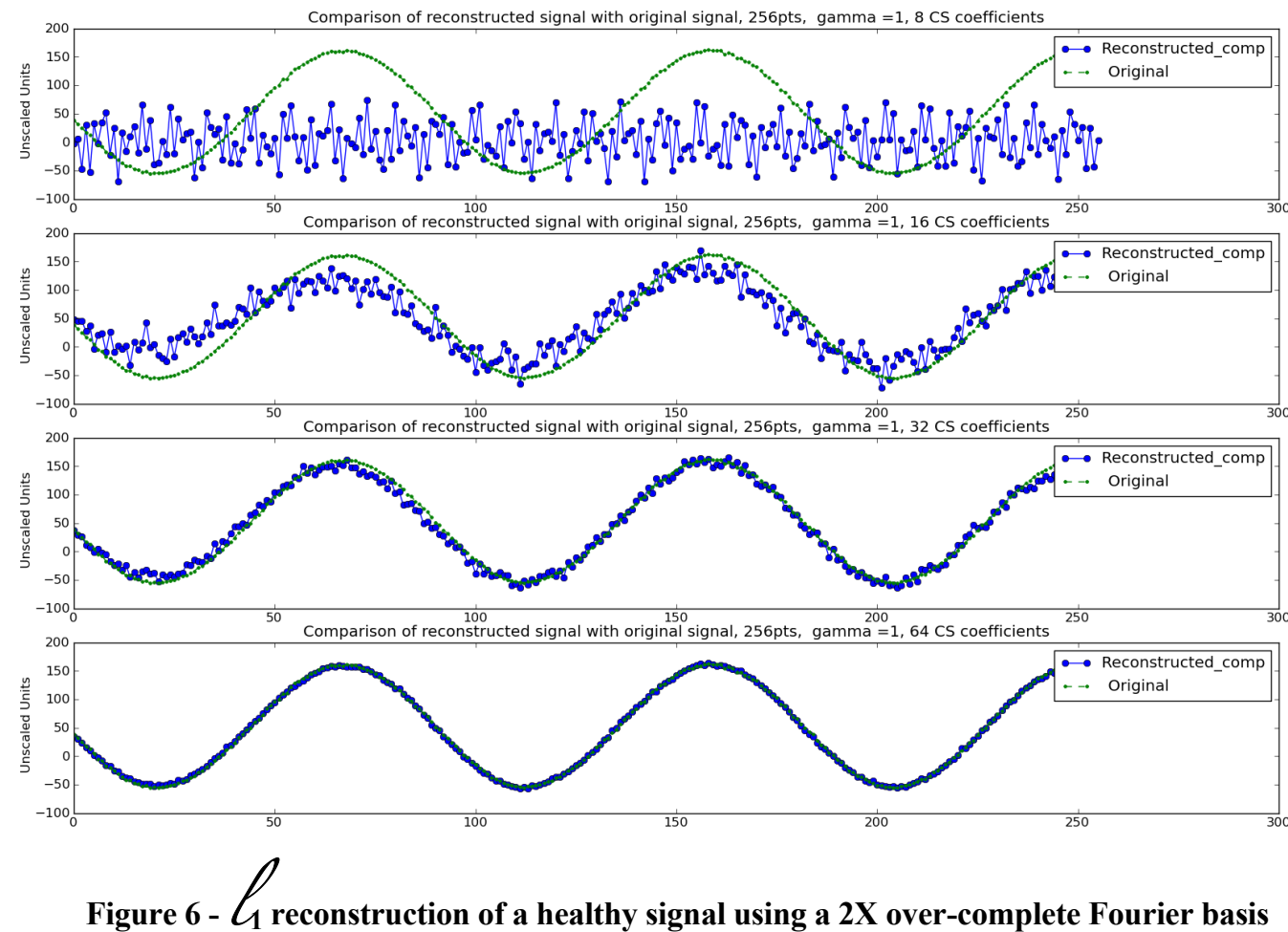


First compare Figure 2 with Figure 6. Clearly, the reconstruction using the overcomplete dictionary outperforms the reconstructions using simply the standard Fourier basis. In this case, when using the over-complete dictionary the performance when using only 16 compressed coefficients is roughly similar to the case when using 32 compressed coefficients with the standard Fourier dictionary. Across all the reconstructions for the healthy case with $L=2$, using the over-complete dictionary produces reconstructions that are comparable to the reconstructions achieved using the standard Fourier basis, but with two times as many compressed measurements. The reconstructions show in Figure 7 show a similar result. Significantly fewer compressed measurements are needed to reconstruct a signal generated by a linear system when an over-complete Fourier basis is used to perform the reconstruction.

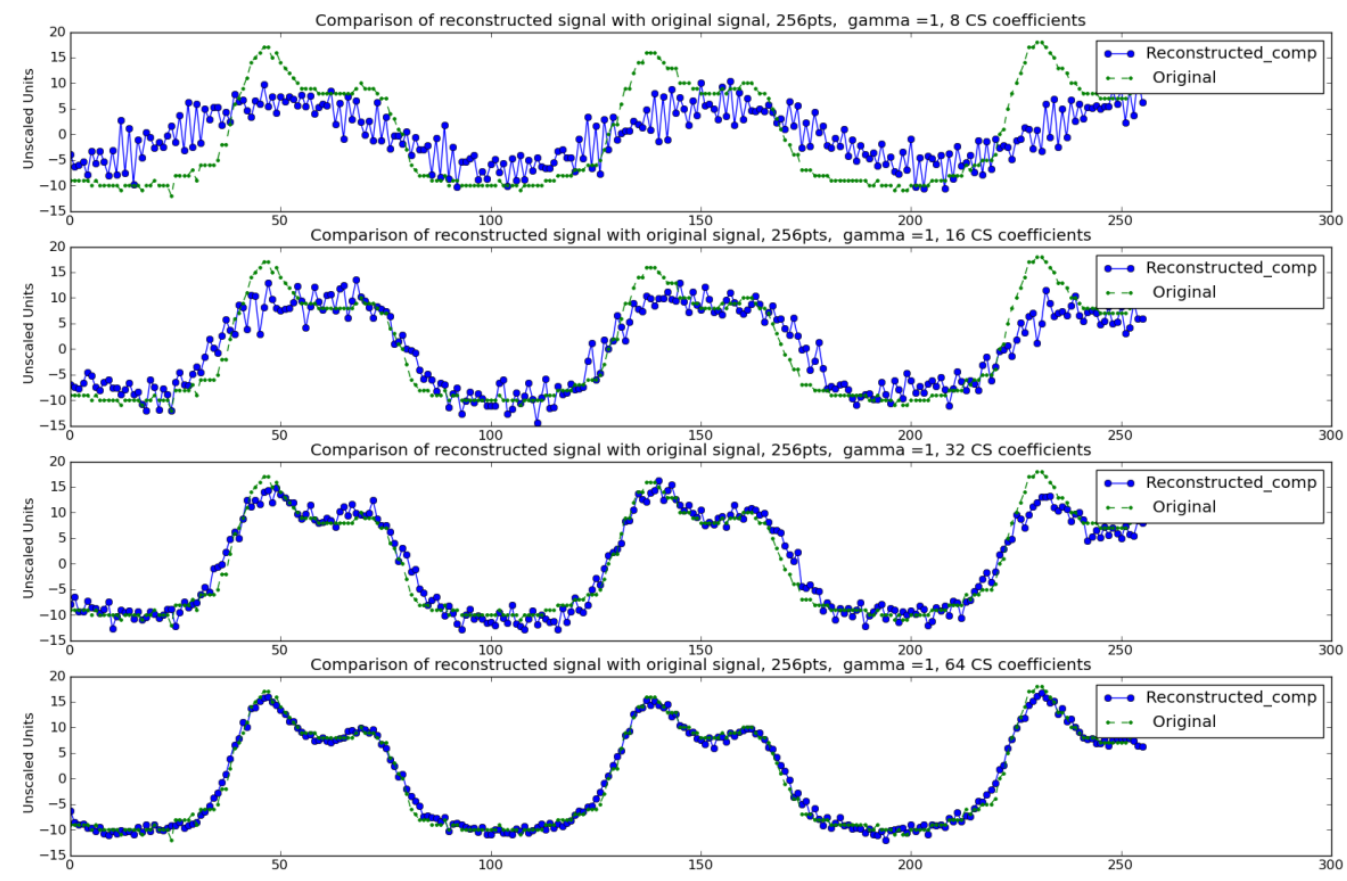

Figure 7 - 4 reconstruction of a damaged signal using a 2X over-complete Fourier basis.

\section{SMASHED FILTER}

In structural health monitoring, the main concern is generally the detection of damage and not necessarily the collection of accurate time series. With this in mind it was decided to investigate alternate techniques to try and detect the presence of damage while using a relatively small number of compressed coefficients. An extension of the matched filter to the compressed domain known as the "smashed filter" seemed an appropriate technique to evaluate [17]. The matched filter is an optimal detection scheme for detecting the presence of a known signal obscured by white Gaussian noise [18]. The implementation is simply:

$$
T(N-1)=\sum_{k=0}^{N-1} h_{n}(k) x(k)
$$

Mascareñas, D., Cattaneo, A., Theiler, J., Farrar, C., Los Alamos National Laboratory, PO Box 1663 MS T001, Los Alamos, NM 87544, LA-UR 12-01484 
Where $x$ is the measured signal, $h_{n}$ is the signal whose presence is being ascertained, $N$ is the length of the signal, and $T(N-1)$ is the test statistic. If the value of the test statistic $T(N-1)$ is above a user-defined threshold, the decision is made that the signal is present in the measurement. The smashed filter is implemented in basically the same manner as the conventional matched filter. The main difference is that the smashed filters are generated by taking time-shifted versions of the signals of interest, and then subjecting them to the measurement process $\Phi$. The time-shifted versions of the signal of interest are used because in this work, the arrival in time of the signal of interest is not known. To compensate a dictionary of time-shifted versions of the signal of interest are used instead. The matched filters in the compressed domain $H_{m}$ for the discrete time-shift $n$ are generated from the signals of interest $h_{n}$ as:

$$
H_{m}=\Phi h_{n}
$$

The smashed filter can then be written as:

$$
T(N-1)=\max _{M} \sum_{k=0}^{m-1} H_{m}(k) y(k)
$$

Where $y$ is the measured signal in the compressed domain, $H_{m}$ is the compressed version of the signal whose presence is being ascertained, $m$ is the number of compressed coefficients, and $T(N-1)$ is the test statistic. For this work, training signals from both the damaged and undamaged states were collected. The training signal for the damaged case was collected at a different time in order to allow the possibility for a small mismatch between the training data and the data acquired from the partially damaged structure. Once the smashed filters were generated, 303 experiments resulting in 128 compressed coefficients per experiment were collected from the structure in both the damaged and healthy cases. Subsets of the 128 compressed coefficients were then used to evaluate the performance of the smashed filter for various numbers of compressed coefficients. To implement the smashed filter, the inner product of the compressed coefficients and the smashed filter vectors was calculated for each experiment. The smashed filter with the largest inner product was then selected and the experiment was classified as damaged or healthy based on whether or not the corresponding smashed filter came from the damaged or healthy case. Table 1 and Table 2 illustrate the results of applying the smashed filter to the experiments for the healthy and damaged cases, respectively. Table 3 shows the numerical results of these experiments. From this data we see that once at least 16 compressed coefficients are used to calculate smashed filters, the probability of misclassifications become very low. No more than 8 in 303 experiments and usually less. It is noteworthy that the number of compressed coefficients needed to achieve accurate classification with the smashed filter is only $1 / 16$ the number of data points in the original time series measurement. Based on these results, the smashed filter has the potential to significantly reduce the number of measurements needed to classify whether or not a structure is damaged.

TABLE 1. SMASHED FILTER RESULTS FOR HEALTHY CASE 


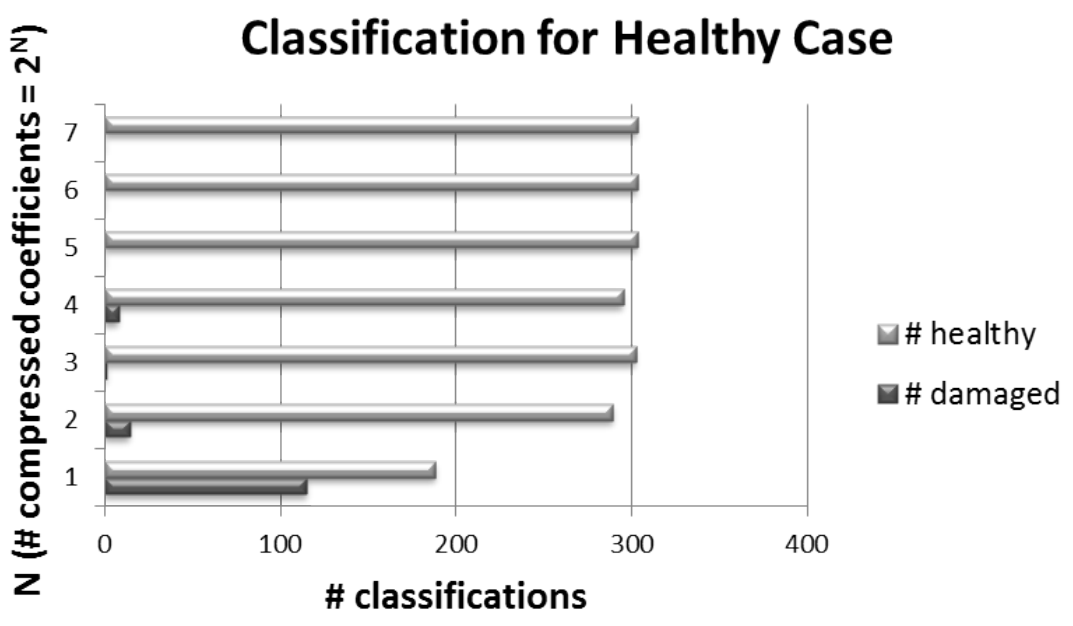

TABLE 2. SMASHED FILTER RESULTS FOR DAMAGED CASE

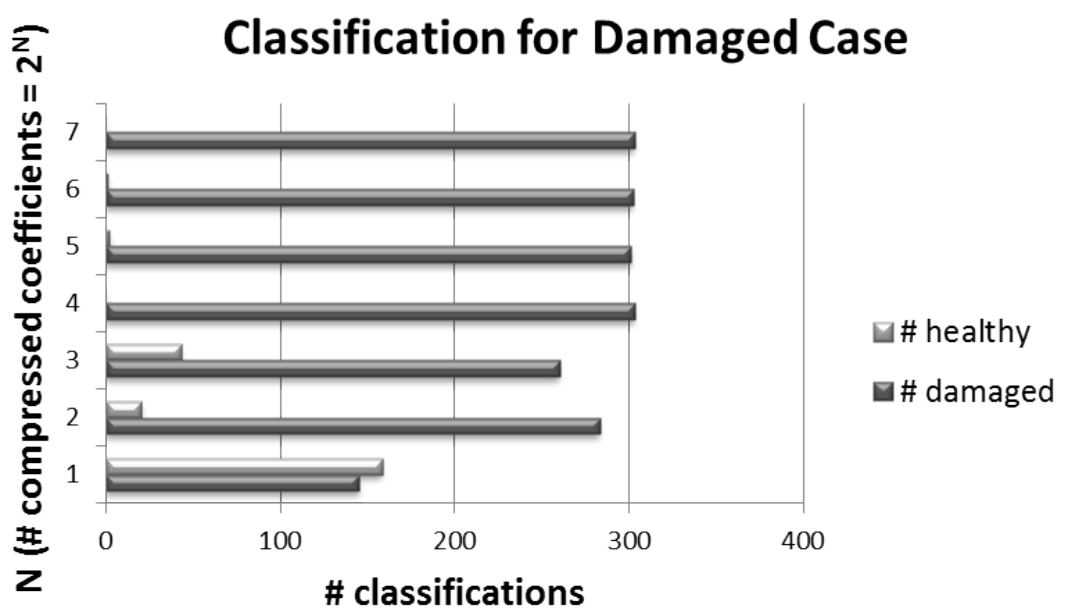

TABLE 3. SMASHED FILTER CLASSIFICATION RESULTS

\begin{tabular}{|c|l|c|c|c|c|c|c|c|}
\cline { 2 - 9 } \multicolumn{1}{c|}{} & \# CS coefficients & $\mathbf{2}$ & $\mathbf{4}$ & $\mathbf{8}$ & $\mathbf{1 6}$ & $\mathbf{3 2}$ & $\mathbf{6 4}$ & $\mathbf{1 2 8}$ \\
\hline \multirow{2}{*}{$\begin{array}{c}\text { Healthy } \\
\text { Case }\end{array}$} & \# damaged & 115 & 14 & 1 & 8 & 0 & 0 & 0 \\
\cline { 2 - 10 } & \# healthy & 188 & 289 & 302 & 295 & 303 & 303 & 303 \\
\hline $\begin{array}{c}\text { Damaged } \\
\text { Case }\end{array}$ & \# damaged & 145 & 283 & 260 & 303 & 301 & 302 & 303 \\
\cline { 2 - 10 } & \# healthy & 158 & 20 & 43 & 0 & 2 & 1 & 0 \\
\hline
\end{tabular}

\section{COMPRESSED SENSING WIRELESS SENSOR NETWORK FRAMEWORK}

The results obtained in this work doing $C_{1}$ norm minimization and using the smashed

filter suggest a signal classification approach that will better enable novel, low-power, low-bandwidth, robust wireless sensor networks. The main power consumer in Mascareñas, D., Cattaneo, A., Theiler, J., Farrar, C., Los Alamos National Laboratory, PO Box 1663 MS T001, Los Alamos, NM 87544, LA-UR 12-01484 
wireless sensor nodes is generally the wireless transmission of data. For this reason, the proposed framework should reduce the amount of data needed for wireless transmission. The proposed framework would have two main processing stages. The first stage would be an extremely low-power smashed filter processor. The smashed filter stage would collect a minimum of coefficients needed to perform adequately reliable classification. The smashed filter is simply a matrix-vector multiplication operation over integers and can be performed on-board the sensor node relatively easily. Alternatively, the very small number of smashed filter coefficients can be transmitted to other sensor nodes or base stations for further analysis. If preliminary smashed filter analysis indicates that the wireless sensor node is experiencing an "event of interest" it may be deemed wise to request and adequate number of compressed coefficients be transmitted to a base-station to perform $\ell_{1}$ norm minimization reconstruction. In order to further minimize the number of required compressed coefficients that must be transmitted, the base-station could make use of dynamic updating techniques for $\ell_{1}$ minimization as described in [19]. In this way the

base-station could perform reconstruction and request more CS measurements if it determines the reconstructed signal is still changing significantly as measurements are added. Once it is deemed that an adequate reconstruction of the signal has been obtained, the techniques developed by the structural health monitoring community can be leveraged to perform detailed analysis on the measurements.

The adoption of a multi-stage compressed sensing framework has the potential to significantly reduce the power and bandwidth requirements of a wireless sensor network for structural health monitoring applications. The further development and analysis of potential compressed sensing wireless sensor network frameworks for structural health monitoring applications will be the focus of future research efforts.

\section{CONCLUSIONS}

The contribution of this work is the development of a 2-stage compressed sensing framework with the potential to conserve energy and bandwidth in embedded wireless sensor nodes for structural health monitoring applications. The fitness of the 2-stage compressed sensing framework for structural health monitoring applications has been shown by executing performance evaluations of $C_{1}$ norm regularization to reconstruct

time series measurements of acceleration collected from representative structures. This work was followed by an evaluation of the smashed filter for damage classification in the same representative structure. It was found that the smashed filter has potential to significantly reduce the number of measurements required to classify the state of health of a structure. The 2-stage compressed sensing framework proposed in this work can readily be applied to embedded wireless sensor networks for structural health monitoring applications with the effect of lowering bandwidth and power requirements. In order to make this system a reality, future research should consider the use of sparse binary measurement matrices $\Phi$ in order to reduce the time

Mascareñas, D., Cattaneo, A., Theiler, J., Farrar, C., Los Alamos National Laboratory, PO Box 1663 MS T001, Los Alamos, NM 87544, LA-UR 12-01484 
and energy needed to apply the digital compressed sensing matrix vector multiplication used to generate the compressed measurements [20]. It is also important to select an appropriate dictionary of signals for the optimization routine to try and find a sparse match too. Ongoing research by the authors is revealing that the choice of the dictionary used to perform the signal reconstruction has a significant effect on the quality of the reconstruction. Preliminary results suggest that the choice of the reconstruction dictionary is application dependent. Another technique that looks very promising for structural health monitoring applications is the SpaRCS procedure for recovering low-rank and sparse matrices from compressive measurement [21]. For many applications, the low-rank portion of this matrix would correspond to the system dynamics, and the sparse portion would correspond to anomalous damage expressing itself in the measurement. This technique would be particularly useful for SHM applications that require full-field measurements, and long-term monitoring of structures for anomalous events.

\section{ACKNOWLEDGEMENTS}

This project was funded by the Los Alamos National Laboratory-Laboratory Directed Research and Development (LDRD) program [20110462ER], 2011. David Mascarenas is also supported by the same program as a Director's Funded Postdoctoral Fellow [20100594PRD1], 2010-2012.

\section{REFERENCES}

1. Lynch JP, Law KH, Kiremidjian AS, Kenny T, Carryer E. A Wireless Modular Monitoring System for Civil Structures. In Proceedings of the 20th International Modal Analysis Conference (IMAC XX); 2002; Los Angeles, CA.

2. Mascarenas D, Flynn E, Farrar C, Park G, Todd M. A Mobile Host Approach for Wireless Powering and Interrogation of Structural Health Monitoring Sensor Networks. IEEE Sensors Journal. 2009; 9(12): p. 1719-1725.

3. Kirolos S, Laska J, Wakin M, Duarte M, Baron D, Ragheb T, et al. Analog-to-information Conversion via Random Demodulation. In IEEE Dallas Circuits and Systems workshop; 2006; Dallas, Texas.

4. Robucci R, Gray JD, Chiu LK, Romberg J, Hasler P. Compressive Sensing on a CMOS SeparableTransform Image Sensor. Proceedings of the IEEE. June 2010; 98(6): p. 1089-1101.

5. Candes E. Compressive Sampling. In Int. Congress of Mathematics; 2006; Madrid, Spain. p. 14331452.

6. Baraniuk R. Compressive sensing. IEEE Signal Processing Magazine. 2007 July: p. 118-121.

7. Candes E. An introduction to compressive sampling. IEEE Signal Processing Magazine. 2008 March: p. 14-20.

8. Candes E, Romberg J, Tao T. Robust Uncertainty principles: exact signal reconstruction from highly incomplete frequency information. IEEE Trans. Inform. Theory. 2006; 52: p. 489-509.

9. Cortial J, Farhat C, Guibas L, Rajashekhar M. Compressed sensing and time-parallel reduced-order modeling for structural health monitoring using a DDDAS. In IEEE Int. Conf. Computational Science (ICCS)); 2007; Beijing, China. p. 1171--1179.

10. Bao Y, Beck J, Li H. Compressive sampling for accelerometer signals in structural health monitoring. Structural Health Monitoring. May 2011; 10(3): p. 235-246.

11. Figueiredo E, Park G, Figueiras J, Farrar C, Worden K. Structural Health Monitoring Algorithm Comparison using Standard Data Sets. Los Alamos National Laboratory technical report; March, 2009. Report No.: LA-14393.

Mascareñas, D., Cattaneo, A., Theiler, J., Farrar, C., Los Alamos National Laboratory, PO Box 1663 MS T001, Los Alamos, NM 87544, LA-UR 12-01484 
12. Nishio M, Hemez F, Worden K, Park G, Takeda N, Farrar C. Feature Extraction for Structural Dynamics Model Validation, Linking Models and Experiments. In Proc. Of the Society for Experimental Mechanics Series; 2011. p. 153-163.

13. Laska J, Kirolos S, Massoud Y, Baraniuk R, Gilbert A, Iwen M, et al. Random sampling for analogto-information conversion of wideband signals. In IEEE Dallas Circuits and Systems Workshop; October 29-30, 2006.2006; Dallas, TX. p. 119 - 122.

14. Mattingly J, Bod S. CVXMOD-Convex optimization software in python. In ; 2008.

15. Harris F. On the Use of Windows for Harmonic Analysis with the Discrete Fourier Transform. Proc. Of the IEEE. January 1978.; 66(1): p. $51-83$.

16. Chen S, Donoho D, Saunders M. Atomic decomposition by basis pursuit. SIAM journal on Scientific Computing. 1998.; 20: p. 33-61.

17. Davenport M, Wakin MDM, Laska J, D. Takhar K, Kelly, Baraniuk R. The smashed filter for compressive classification and target recognition. In Proc. IS\&T/SPIE Symp. Elec. Imag.: Comp. Imag.. January 2007..

18. Kay S. Fundamentals of Statistical Signal Processing. 1st ed.: Prentice Hall; 1998.

19. Asif MS, Romberg J. Dynamic updating for L1 minimization. IEEE Journal of selected topics in signal processing. April 2010;: p. 421 - 434.

20. Berinde R, Gilbert AC, Indyk P, Karloff H, Strauss MJ. Combining geometry and combinatorics: A unified approach to sparse signal recovery. In 46th Annual Allerton Conference on Communication, Control, and Computing; 2008; Urbana-Champaign, IL. p. 798-805.

21. Waters AE, Sankaranarayanan AC, Baraniuk RG. SpaRCS: Recovering Low-Rank and Sparse Matrices from Compressive Measurements. In Neural Information Processing Systems (NIPS); 2011; Granada, Spain. p. 1089-1097. 